\title{
Distribution of Retardation Times from the Photon Correlation Spectra of Glass Forming Systems
}

\author{
Dedicated to the Memory of the late Professor Ichiro Sakurada \\ J.-U. Hagenah, G. Meier, G. Fytas,* \\ and E. W. FISCHER \\ Max-Planck-Institut für Polymerforschung, \\ Postfach 3148, 6500 Mainz, BRD
}

(Received January 22, 1987)

\begin{abstract}
For viscoelastic molecular and macromolecular systems near and above the glass transition temperature the distribution of retardation times $L(\log \tau)$ can be determined by using the dynamic light scattering spectrum arising from slow density fluctuations. We use the CONTIN inverse Laplace transform (ILT) technique to extract the $L(\log \tau)$ of the time correlation functions of pentachlorobiphenyl, poly(vinyl acetate) and poly(methyl methacrylate). For cases where the correlation function can be described by a single Kohlrausch-Williams-Watts (KWW) fractional exponential decay function the results obtained from numerical Laplace inversion of the KWW function and the ILT technique are identical. For poly(methyl methacrylate) however, the ILT technique seems to reveal more clearly the underlying structure of the correlation functions.
\end{abstract}

KEY WORDS Light Scattering / Photon Correlation Spectroscopy /

Retardation Times Distribution / Glass Forming Systems / Polymers /

Photon correlation spectroscopy is being extensively used to measure diffusion coefficients for macromolecular solutions where light scatters mainly by concentration fluctuations. ${ }^{1,2}$ For polydisperse systems, molecular weight and size particle distributions can be obtained from the photon correlation functions of high precision. ${ }^{3}$ A comparison of the deconvolution techniques applied in this context is recently reported. ${ }^{4}$ So far, much less attention have received these techniques in the case of density autocorrelation functions measured in neat molecular liquids and bulk amorphous polymers near and above the glass transition temperature $\left(T_{\mathrm{g}}\right)$. Instead, the KohlrauschWilliams-Watts (KWW) fractional exponential decay is used to represent the highly nonexponential time correlation functions of density fluctuations. ${ }^{5}$ Clearly the KWW representation had no physical basis for a long time. In the last years attempts have been made to change this. ${ }^{19,20}$

The correlation functions of light isotropically scattered by bulk polymers near $T_{\mathrm{g}}$ measure the time dependent longitudinal compliance. ${ }^{6}$ In the first experimental verification, the distribution of retardation times (DRT) evaluated from the time correlation functions of poly(vinyl acetate) ${ }^{7}$ was found to be similar to that of the bulk compliance. ${ }^{8}$ The extraction of the distribution of retardation times from the correlation functions using inverse Laplace transform (ILT) was further pursued for oligomeric polystyrene ${ }^{9}$ and high molecular

* Permanent address: Department of Chemistry, University of Crete and Research Center of Crete, Iraklion Crete, Greece. 
weight poly(methyl methacrylate) ${ }^{10}$ For the latter polymer, the DRT clearly revealed two relaxation modes also observed by mechanical and dielectric relaxation experiments.

In this article, we apply an Inverse Laplace Transformation (ILT) technique (referred to as CONTIN) originally developed by Provencher ${ }^{11}$ and slightly modified to evaluate the DRT of viscoelastic bulk systems. This procedure is carried out without an a priori assumption of the shape of DRT in contrast to the fit of the data to the KWW function. The modified ILT technique is first tested using computer generated data and then applied to the experimental correlation functions of the molecular glass pentachlorobiphenyl $\left(\Phi \Phi \mathrm{Cl}_{5}\right)$ for both polarized and depolarized scattering, poly(vinyl acetate) (PVAc) and poly(methyl methacrylate) (PMMA). The correlation functions were measured over 4.3 decades in one run with a logarithmic spaced delay time correlator.

\section{METHODS OF COMPUTATION}

The desired normalized correlation function $C(t)$ of the scattered electric field is related to the measured intensity autocorrelation function $G(t)$ through the Siegert relation:

$$
G(t)=A\left(1+f \cdot a^{2}|C(t)|^{2}\right)
$$

where $A$ is the baseline calculated or measured at long delay times $t$. The instrumental factor $f$ depends mainly on the size and distance of pinholes determining the spatial coherence and the factor $a$ is the fraction of the total scattered intensity (arising from slow density fluctuations). In this case, $C(t)$ is identified with the autocorrelation function

$$
C(t)=\frac{\left\langle\varepsilon_{\mathrm{q}}(t) \varepsilon_{\mathrm{q}}(0)\right\rangle}{\left\langle\left|\varepsilon_{\mathrm{q}}(0)\right|^{2}\right\rangle}
$$

where $\varepsilon_{\mathrm{q}}(t)$ is the Fourier transform of the dielectric constant fluctuation $\delta \varepsilon(r, t) . C(t)$ is generally well represented ${ }^{5}$ by the $\mathrm{KWW}$ equation.

$$
C(t)=\exp \left[-\left(t / \tau_{0}\right)^{\beta}\right]
$$

where $\beta(0<\beta \leq 1)$ is a measure of the width of the distribution of retardation times. In most cases, $\beta>0.3$ and this approach yields a physically meaningful average relaxation time

$$
\bar{\tau}=\left(\tau_{0} / \beta\right) \cdot \Gamma\left(\beta^{-1}\right)
$$

with $\Gamma\left(\beta^{-1}\right)$ being the gamma function.

Alternatively, like in mechanical relaxation experiments $C(t)$ can be represented by a multiexponential decay given by ${ }^{12}$

$$
C(t)=\int_{0}^{\infty} \mathrm{e}^{-t / \tau} \rho(\tau) \mathrm{d} \tau
$$

where $\rho(\tau)$ is a distribution function. A more common expression for $C(t)$ is

$$
C(t)=\int_{-\infty}^{\infty} \mathrm{e}^{-t / \tau} L(\ln \tau) \mathrm{d} \ln \tau
$$

with $L(\ln \tau)=\tau \cdot \rho(\tau)$ being the distribution of retardation times usually obtained from compliance measurements. ${ }^{12}$ However, since the KWW equation 3 represents in most cases well the experimental time correlation functions we first give the $L^{\prime}(\ln \tau)$ corresponding to the KWW function. ${ }^{13}$

$$
L^{\prime}(\ln \tau)=\frac{1}{\pi \chi} \int_{0}^{\infty} \mathrm{e}^{-x u} \mathrm{e}^{-u^{\beta} \cos \pi \beta} \sin \left(u^{\beta} \sin \pi \beta\right) \mathrm{d} u
$$

where $\chi=\tau / \tau_{0}$. For the long relaxation time region $(\chi>1)$ we use the expression recently given by Helfand. ${ }^{14}$ Provencher ${ }^{11}$ has developed a computer algorithm to extract $\rho(\tau)$ from $C(t)$ of polydisperse macromolecular solutions. This is a constrained ILT in the sense that besides the least-squares the program also considers the sum of the second derivatives of $\rho(\tau)$ multiplied by the constraining factor. The latter lies in the range $10^{-8}$ to $10^{-1}$. We have slightly modified the original CONTIN program to yield $L(\ln \tau)$. To check our modification, we have also evaluated $\rho(\tau)$ in the case of undiluted $\Phi \Phi \mathrm{Cl}_{5}$. Figure 1 shows $\tau \rho(\tau)$ 


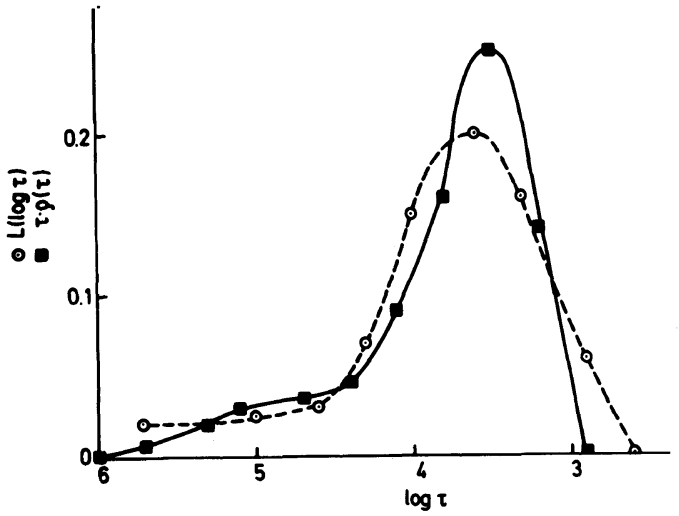

Figure 1. Comparison of distributions of retardation times obtained by two different numerical evaluation methods: Original CONTIN program giving $\tau \cdot \rho(\tau)$. Modified CONTIN program giving $L(\log \tau)$. Original correlation function was from $\Phi \Phi \mathrm{Cl}_{5}$ at $270 \mathrm{~K}$.

and $L(\log \tau)$ obtained from the inversions of $(G(t) / A-1)^{1 / 2}$ at $270 \mathrm{~K}$ using the original and modified CONTIN algorithm respectively. This plot suggests that the distributions expressed in $\tau \rho(\tau)$ and $L(\log \tau)$ are experimentally the same. Hereafter, we use the expressions $L(\log \tau)$ for the distribution of retardation times. The amplitude $b=\left(f a^{2}\right)^{1 / 2}$ and the mean $\langle\log \tau\rangle$ are respectively given by the zeroth and first moment of the distribution $L(\log \tau)$.

$$
\begin{aligned}
b & =\int_{-\infty}^{\infty} L(\log \tau) \mathrm{d} \log \tau \\
\langle\log \tau\rangle & =\int_{-\infty}^{\infty}(\log \tau) L(\log \tau) \cdot \mathrm{d} \log \tau
\end{aligned}
$$

On the other hand, the variance $\sigma$ of the $L(\log \tau)$ is evaluated from

$$
\sigma=\int_{-\infty}^{\infty}(\log \tau-\langle\log \tau\rangle) L(\log \tau) \mathrm{d} \log \tau
$$

\section{EXPERIMENTAL}

The samples used are given in Table I. A dust free pentachlorobiphenyl $\left(\Phi \Phi \mathrm{Cl}_{5}\right)$ sample was prepared by filtration at about $50^{\circ} \mathrm{C}$
Table I. Molecular weight and glass transition temperatures of the amorphous samples

\begin{tabular}{lccl}
\hline Sample & $T_{\mathrm{g}} / \mathrm{K}$ & $M$ & \multicolumn{1}{c}{ Supplier } \\
\hline$\Phi \Phi C C_{5}$ & 251 & 324 & Bayer \\
PVAc $^{\mathrm{a}}$ & 290 & $15000\left(\bar{M}_{n}\right)$ & Wacker Chemie \\
PMMA $^{\mathrm{b}}$ & 323 & $3900\left(\bar{M}_{n}\right)$ & Röhm \\
\hline
\end{tabular}

a PVAc, poly(vinyl acetate).

b PMMA, poly(methyl methacrylate).

through a $0.45 \mu \mathrm{m}$ Millipore filter into the dust free light scattering cell. To prepare the poly(vinyl acetate) (PVAc) and poly(methyl methacrylate) (PMMA) samples that are suitable for photon correlation measurements we have used the procedure described elsewhere. ${ }^{16}$ A strong confirmation of the suitability of the polymer samples was provided by the low value of the Landau-Plazcek intensity ratio.

The experimental photon correlation functions $G(t)$ at different temperatures were taken at a scattering angle of $90^{\circ}$. The light source was an argon ion laser operating at $\lambda=514.5 \mathrm{~nm}$ with a power of about $400 \mathrm{~mW}$. For the polarized spectra both the incident and scattered light were polarized vertically $(V)$ with respect to the scattering plane. On the other hand, the depolarized spectrum of $\Phi \Phi \mathrm{Cl}_{5}$ was recorded with laser light polarized parallel $(H)$ to the scattering plane. The scattered light was filtered by a narrow band interference filter and detected by the photomultiplier tube (EMI). The digital output signal from the fast amplifier-discriminator was plugged into a 28 channel Malvern Log Lin correlator (K-7027) measuring the single clipped autocorrelation function $G(t)$ of the scattered intensity over 4.3 decades in time in one run.

The wide time range covered by the Log $L$ in correlator in one run is sufficient to study relaxation processes with moderate width of distributions $(\beta>0.3)$. However, in the case of poly(alkyl methacrylates), where there is a strong evidence that more than one relaxation process contributes to $G(t)$ a time range larger than 4.3 decades is required. ${ }^{10,15,16}$ Thus for 
the PMMA sample partially overlapping correlograms were spliced together using the matching procedure described elsewhere. ${ }^{17}$

The ILT program was kindly provided by Dr. Provencher (EMBL, Heidelberg) and run on an Honeywell-Bull (HB 66) computer. After representing the experimental net correlation function $(G(t) / A-1)^{1 / 2}=b C(t)$ with the $\mathrm{KWW}$ equation 3 , the corresponding distribution $L^{\prime}(\log \tau)$ was numerically evaluated via eq 7. Alternatively, the distribution $L(\log \tau)$ was obtained by fitting eq 6 to the experimental $b \cdot C(t)$ using the CONTIN routine. A confidence criterion for the fit, along with the residual plot, is the value of the constraining factor. ${ }^{11}$

\section{RESULTS}

\section{Test of CONTIN with Computer Generated Data}

First we tested the correctness of the procedure to get the distribution $L^{\prime}(\log \tau)$ corresponding to the $\mathrm{KWW}$ eq 3 . To do so, we have numerically evaluated $L^{\prime}(\log \tau)$ for three values of $\beta(1 / 3,1 / 2,2 / 3)$ using eq 7 in the region $\chi<1$ and Helfand's expression ${ }^{14}$ for $\chi>1$. For these $\beta$ values, the $L^{\prime}(\log \tau)$ can be expressed in closed analytical forms. ${ }^{14}$ The two distributions thus obtained are found to be identical.

Since the KWW equation generally fits well the experimental data, we used KWW functions to test the CONTIN program. Figure $2 \mathrm{a}$ displays two KWW functions with $\tau_{0}=$ $3.10^{-3}$ s and $\beta=0.65$ and 0.35 which are typical distribution parameters found in molecular $^{18}$ and polymeric ${ }^{5,7}$ glass forming systems. The average time $\bar{\tau}$ (eq 4) amounts to $4.1 \times 10^{-3} \mathrm{~s}$ and $15 \times 10^{-3} \mathrm{~s}$ for $\beta$ equal to 0.65 and 0.35 , respectively. The distribution $L^{\prime}(\log \tau)$ (eq 7) corresponding to the KWW function is compared to the $L(\log \tau)$ obtained from the inversion of the $\mathrm{KWW}$ function using the CONTIN routine. The excellent agreement between the two distribu- tions for both $\beta$ values is visualized in Figure 2b. The variance $\sigma$ computed from eq 19 amounts to 2.1 and 7.7 for the narrow and broad $L(\log \tau)$ respectively, whereas the corresponding $\langle\log \tau\rangle$ (eq 9) are -2.65 and -2.81 . In contrast to the average time $\bar{\tau}$, the mean $\langle\log \tau\rangle$ is less sensitive to the value of $\beta$ in this range. Finally, for the broad distribution the amplitude $b$ in eq 8 is $3.5 \%$ smaller than the chosen value.

\section{Molecular Glass Forming Systems}

We consider next the photon correlation functions of $\Phi \Phi \mathrm{Cl}_{5}$ at $270 \mathrm{~K}$ for both polarized and depolarized scattering. Since the latter involves only fluctuations in the optical anisotropy, ${ }^{1}$ in contrast to the polarized light scattering involving density fluctuations as well, we expect slightly narrower distributions $L_{\mathbf{V H}}(\log \tau)$ for the depolarized component of the scattered light. ${ }^{18}$ As mentioned previously, we handle the data in the form of the net correlation function $(G(t) / A-1)^{1 / 2}$ i.e., using a fixed baseline $A$ in eq 1 . Frequently in the literature, ${ }^{5}$ the analysis of the photon correlation functions of undiluted systems involves a floating baseline which can affect the shape of the DRT.

To examine this effect, we have applied the CONTIN ILT program to the same $G(t)$ data taken in the $V H$ scattering geometry using, however, two values of $A$ that differ by $0.3 \%$. This is a typical error of the baseline $A$ for density correlation functions. The two $L_{\mathbf{V H}}(\log \tau)$ thus obtained are shown in Figure 3. The mean $\langle\log \tau\rangle=-3.52$ and the variance $\sigma=2.1$ are affected by less than $1 \%$. The amplitude $b$ amounts to $0.57 \pm 0.02$ and seems to be insensitive to the baseline variation in this range. For comparison, the KWW representation of the experimental data yields: $\tau_{0}=4.2 \times 10^{-4} \mathrm{~s}, \beta=0.64$, and $\tau_{0}=4.4 \times 10^{-4} \mathrm{~s}$, $\beta=0.62$, respectively for the fit with higher and lower baseline. Hence, the computed average times $\bar{\tau}$ from eq 4 differ by $9 \%$. On the other hand, we conclude from the data of Figure 3 


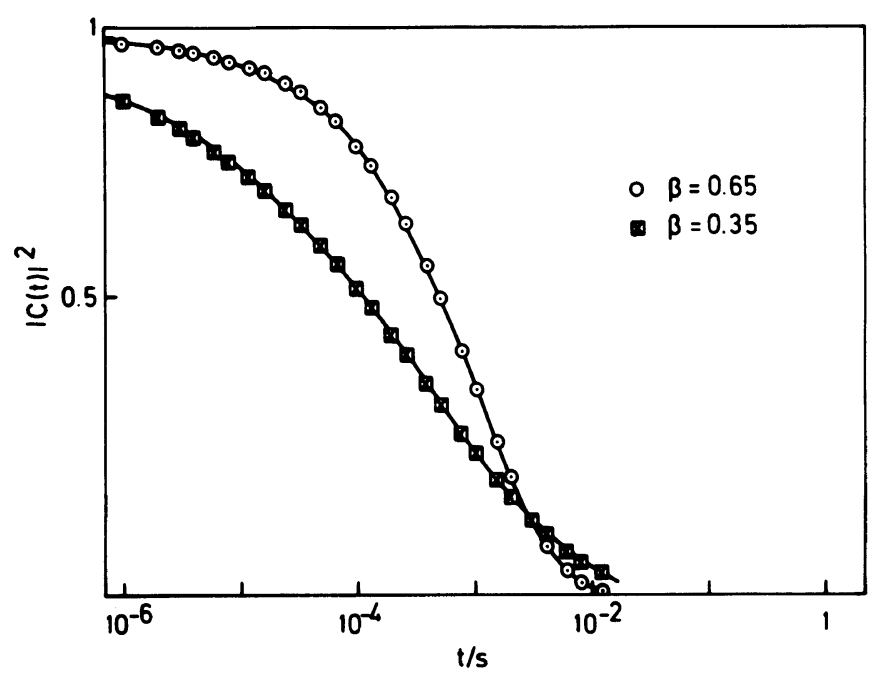

(a)

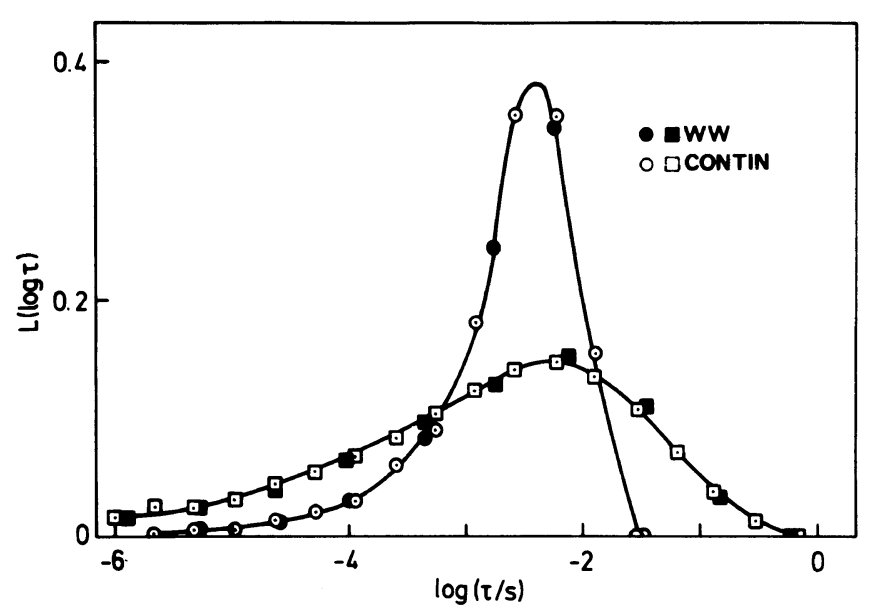

(b)

Figure 2. Computer simulation with $\tau_{0}=3 \times 10^{-3} \mathrm{~s}$ and different values of given $\beta$. (a) Correlation functions using KWW (eq 3). (b) Laplace inversion using CONTIN (hollow symbols) and using eq 7 (solid symbols).

that the typical uncertainty of $0.3 \%$ in the baseline introduces minor changes in the $L(\log \tau)$. Conversely, the latter may be used to index small changes in the shape of the relaxation function $C_{\mathrm{vv}}(t)$ recorded for the polarized component of the scattered light.

Figure 4a displays the experimental function $b^{2}\left|C_{\mathrm{Vv}}(t)\right|^{2} v s$. $\log t$ for $\Phi \Phi \mathrm{Cl}_{5}$ at $270 \mathrm{~K}$. On the other hand, Figure $4 \mathrm{~b}$ shows the $L_{\mathrm{Vv}}(\log \tau)$ obtained from the inversion of the relaxation function yielding $b=0.54, \sigma=2.5$, and $\langle\log \tau\rangle=-3.33$. These values render the expected behavior for the two scattering geometries. $^{18}$ Alternatively, the $\mathrm{KWW}$ fit to the same experimental function leads to $b=0.55, \beta=0.89$, and $\tau_{0}=5.9 \times 10^{-4} \mathrm{~s}$ which further gives $\bar{\tau}=9.1 \times 10^{-4} \mathrm{~s}$. Here, the difference between $\bar{\tau}_{\mathbf{V v}}$ and $\bar{\tau}_{\mathbf{V H}}$ exceeds the error bounds and its therefore significant. Thus, for moderately broad distribution $(\beta>0.3)$ 


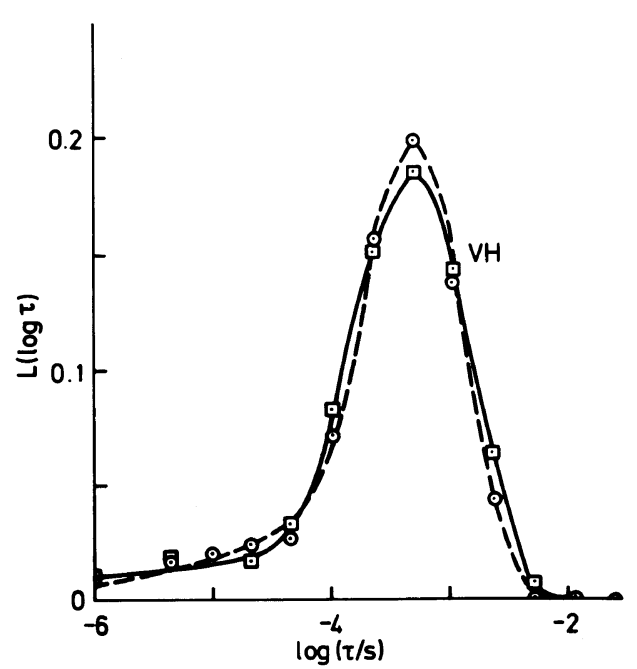

Figure 3. Distribution functions $L(\log \tau)$ obtained with CONTIN using baselines differing by $0.3 \%$. The dashed and solid lines correspond to the lower and higher baseline fit for the depolarized $\left(V_{\mathrm{H}}\right)$ correlation function of $\Phi \Phi \mathrm{Cl}_{5}$ at $270 \mathrm{~K}$.

the ILT of the relaxation function $C(t)$ yields as much information as the $\mathrm{KWW}$ representation.

\section{Amorphous Bulk Polymers}

Photon correlation measurements of PVAc in the temperature range $305 \mathrm{~K}$ to $338 \mathrm{~K}$ have been recently carried out. ${ }^{7}$ Since the depolarized scattering intensity from. PVAc is small, the polarized component measures directly the density correlation function. This was represented well by eq 3 with $b=0.40 \pm 0.02$ and $\beta=0.36 \pm 0.02$ virtually temperature independent over the considered temperature range. The times $\tau_{0}$ and $\bar{\tau}$ which depend strongly on temperature amount respectively to $1.5 \times 10^{-2} \mathrm{~s}$ and $7.3 \times 10^{-2} \mathrm{~s}$ at $313 \mathrm{~K}$. Furthermore, good agreement was found between the light scattering and dynamic compressibility data using certain approximation. ${ }^{6}$ Here we refer to these data in order to extract the distribution of retardation times $L(\log \tau)$ directly from the measured correlation function $G(t)$ using the time range of the $\log L$ in correlator in one run. This situation corre- sponds to the computer generated WW function with $\beta=0.35$ discussed in section of Test of CONTIN with Computer Generated Data.

The normalized correlation function $|C(t)|^{2}$ of PVAc at $313 \mathrm{~K}$ plotted $v s . \log t$ in Figure 5a. The amplitude $b=0.37$ and $\langle\log \tau\rangle=-1.96$ are obtained from eq 8 and 9, respectively. The distribution $L(\log \tau)$ shown in Figure $5 b$ exhibits a single peak whose shape is similar to the retardation time spectrum obtained from mechanical compliance data. ${ }^{6}$ This agreement constitutes a support of the performed data analysis.

For the experimental correlation functions we have so far considered, the time range of the 4.3 decades covered by the commercially available $\log L$ in correlator in one run is sufficient to monitor the relaxation process. For PMMA however, a time range larger than 4.3 decades is required to assure reliably both the amplitude $b$ at short times and baseline $A$ at long times. The matching of partially overlapping correlograms is so far the only applicable procedure. Figure 6 visualizes this situation for PMMA at $338 \mathrm{~K}$. The KWW fit yields $\beta=0.16$ which is found to change significantly with temperature (from 0.26 at $371 \mathrm{~K}$ to 0.16 at $338 \mathrm{~K}$ ). This small and temperature dependent $\beta$, which is in contrast to the usual situation of constant $\beta$ greatly increases the average time $\bar{\tau}(=1200 \mathrm{~s}$ at $338 \mathrm{~K})$ and hence leads to significant distortion of its temperature dependence. An explanation for the relatively small and temperature dependent value of $\beta$ could be the existence of more than one relaxation processe exhibiting different temperature dependences. ${ }^{10}$ However, the correlation function of Figure 6 a described with an apparent $\beta=0.16$ still shows a single distributed relaxation process appart from small systematic derivations which show up in a residual plot when comparing single $\mathrm{KWW}$ with double $\mathrm{KWW}$ representation. ${ }^{16}$

The present method of data analysis which does not assume any functional form of the 


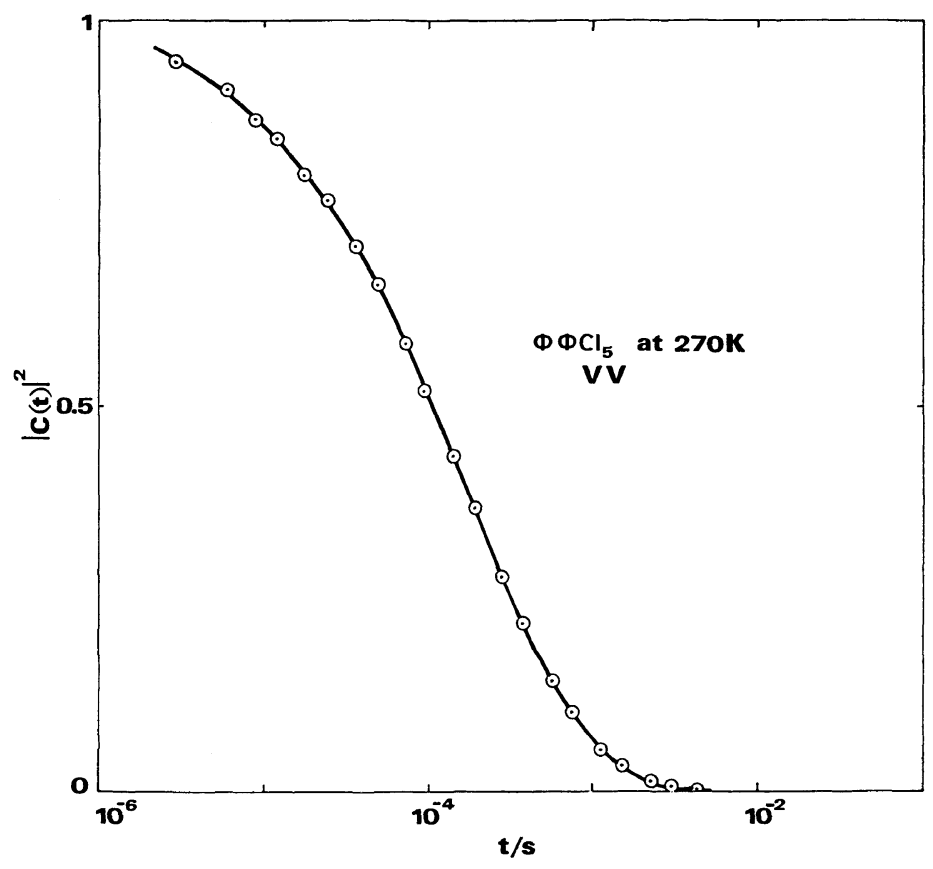

(a)

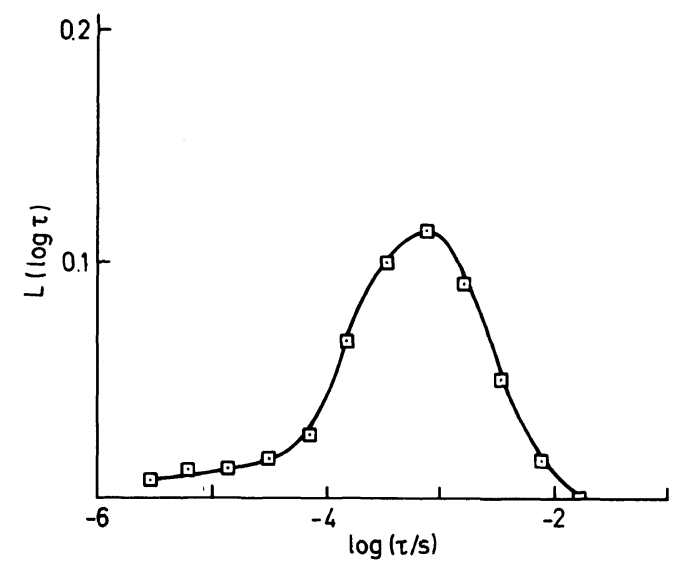

(b)

Figure 4. Polarized $\left(V_{\mathrm{v}}\right)$ component of scattered light from $\Phi \Phi \mathrm{Cl}_{5}$ at $270 \mathrm{~K}$. (a) Net correlation function $b^{2} \cdot|C(t)|^{2}$. (b) Corresponding distribution function $L(\log \tau)$ obtained with CONTIN.

relaxation functions other than that of eq 6 yields the $L(\log \tau)$ of Figure $6 \mathrm{~b}$. Clearly, the $L(\log \tau)$ displays two peaks with the short time peak located at about $10^{-3} \mathrm{~s}$ and the long time peak at about $1 \mathrm{~s}$. The corresponding correlation function (Figure 6a) exhibits no clear kink probably owing to the large width of the distribution of retardation times. Alternatively, the single $\mathrm{KWW}$ fit of this function indeed shows a systematic deviation in a residual plot suggesting an additional relaxation process. The presence of the two re- 


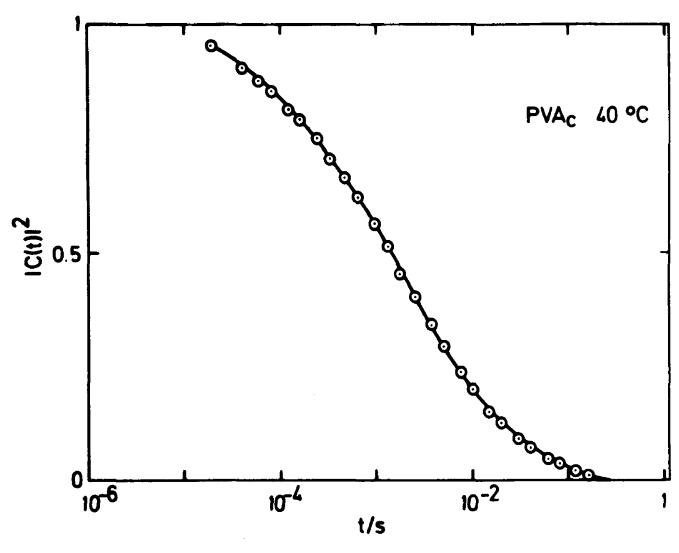

(a)

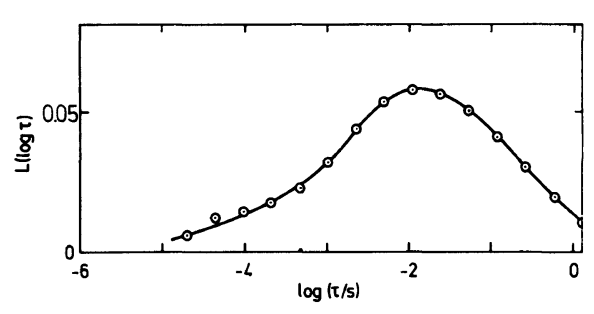

(b)

Figure 5. Poly(vinyl acetate) at $313 \mathrm{~K}$. (a) Normalized correlation function $|C(t)|^{2}$, (b) Corresponding distribution function $L(\log \tau)$ computed using CONTIN.

laxation processes in the correlation function in unambiguously shown in Figure $6 \mathrm{~b}$ for temperatures up to $80^{\circ} \mathrm{C}$. At higher temperature the two processes tend to merge as in the case of high molecular weight PMMA. ${ }^{10}$ Thus the use of the ILT technique to analyse the photon correlation functions with large width of retardation time distributions seems to reveal more clearly the underlying structure than the usual fractional exponential approach of eq 3 , which moreover is not sufficient to represent the experimental data.

\section{CONCLUSION}

The CONTIN ILT routine developed by Provencher mainly for polydisperse macromolecular solutions, was used to extract the distribution of retardation times in the photon correlation functions of undiluted glass forming systems. The experimental density correlation functions were measured using a Log Lin Malvern correlator covering 4.3 decades in time in one run. The CONTIN program first was tested using computer generated data and then used to yield the distribution $L(\log \tau)$ in systems with vastly different width of distributions. Though, however, the ILT of experimental data taken over a finite time range is ill-conditioned the agreement with findings of other experimentals techniques constitutes an additional support of the pursuing data analysis.

\section{REFERENCES}

1. B. Berne and R. Pecora, "Dynamic Light Scattering," Wiley, New York, N.Y., (1976).

2. S. H. Chen, B. Chu, and R. Nossal, (Ed.), "Scattering Techniques Applied to Supramolecular and Nonequilibrium Systems," Plenum Press, New York, N. Y., 1981.

3. B. Dahneke (Ed.), "Measurement of Suspended Particles by Quasi-Elastic Light Scattering," Wiley, New York, N.Y., 1983.

4. R. S. Stock and W. H. Ray, J. Polym. Sci., Polym. Phys. Ed., 23, 1393 (1985).

5. G. D. Patterson, "Photon Correlation Spectroscopy in Advances in Polymer Science, Vol. 48, Springer, Berlin, 1983.

6. C. H. Wang and E. W. Fischer, J. Chem. Phys., 82, 632 (1985).

7. G. Fytas, C. H. Wang, G. Meier, and E. W. Fischer, Macromolecules, 18, 1492 (1985).

8. J. E. Mc Kinney and H. V. Belcher, J. Res. Natl. Bur. Stand., 67A, 43 (1963).

9. P. J. Carroll and G. D. Patterson, J. Chem. Phys., 82, 9 (1985).

10. G. Fytas, C. H. Wang, E. W. Fischer, and K. Mehler, J. Polym. Sci., Polym. Phys. Ed., (1986).

11. S. W. Provencher, Computor Phys. Commun., 27, 213 (1982).

12. J. D. Ferry, "Viscoelastic Properties of Polymers," 
Retardation Time Distribution from PCS

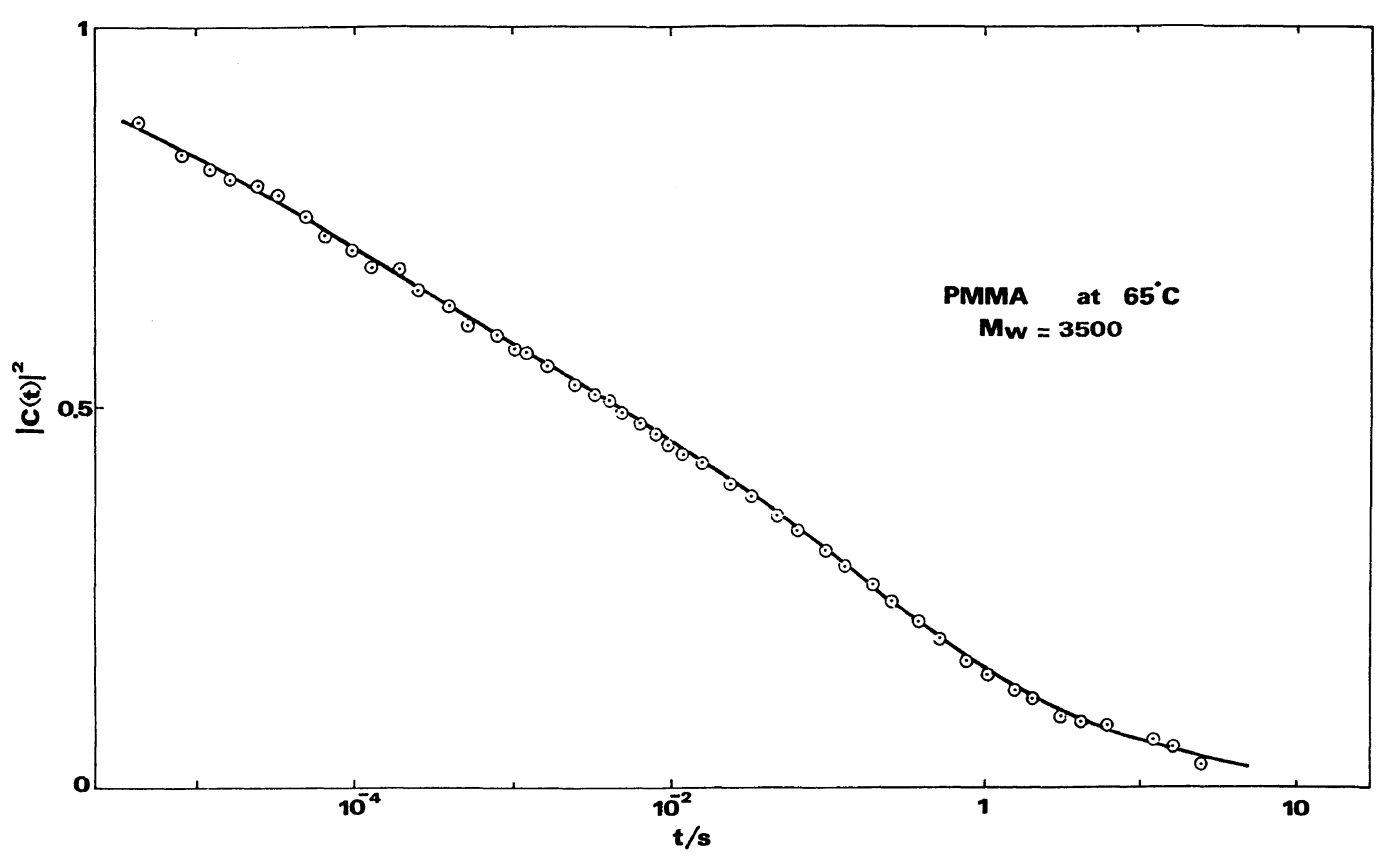

(a)

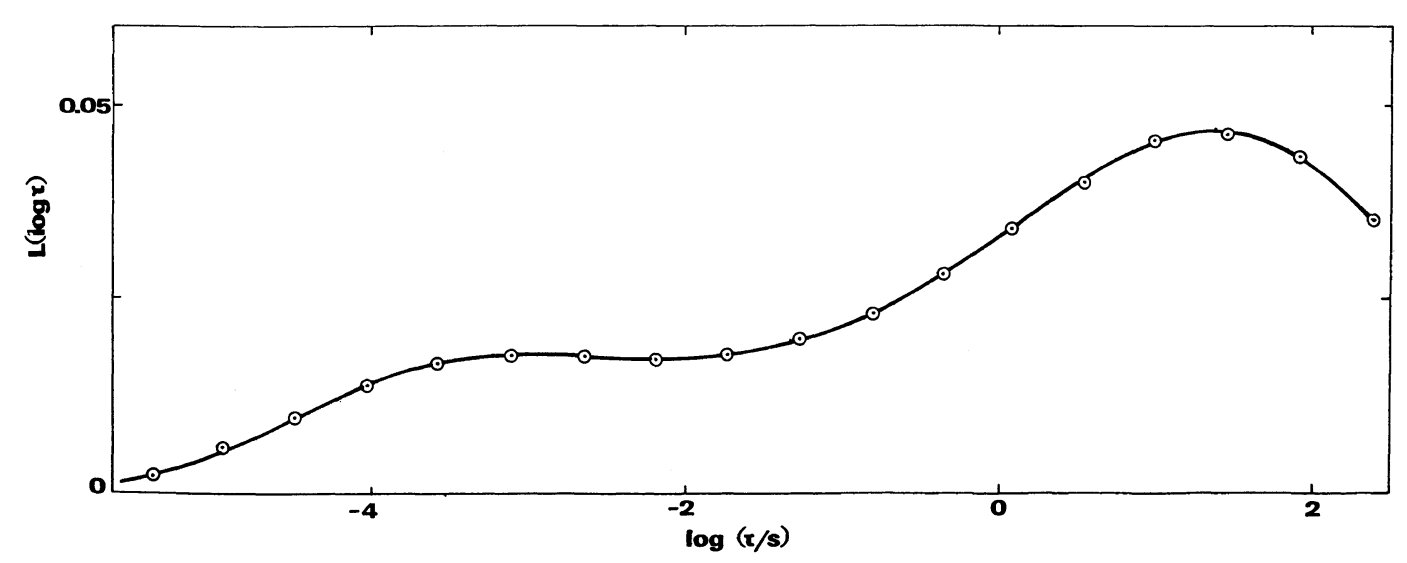

(b)

Figure 6. Poly(methyl methacrylate) at $338 \mathrm{~K}$. (a) Normalized correlation function $|C(t)|^{2}$. (b) Corresponding distribution function $L(\log \tau)$ computed using CONTIN.

3rd ed., Wiley, New York, N.Y., 1980.

13. H. Pollard, Bull. Am. Math. Soc., 52, 908 (1946).

14. H. Helfand, J. Chem. Phys., 78, 1931 (1983).

15. G. D. Patterson, J. R. Stevens, and C. P. Lindsey, J. Macromol. Sci-Phys, B18, 649 (1980).

16. G. Meier, G. Fytas, and Th. Dorfmüller, Macromolecules, 17, 957 (1984).

17. C. H. Wang, G. Fytas, D. Lilge, and Th. Dorfmüller,
Macromolecules, 14, 1363 (1981).

18. G. Fytas, C. H. Wang, D. Lilge, and Th. Dorfmüller, J. Chem. Phys., 75, 4247 (1981).

19. U. L. Ngai, Comments Solid State Phys., 9, 127 (1979); 9, 141 (1980).

20. A. Blumen, J. Klafter, and G. Zumofen, "Fractals in Physics, L. Pietronero and E. Tosatti, Ed., Elsevier Science Publishers, Amsterdam, 1986, p 399. 